\title{
A Technology Management System for the Development of Single-Task Construction Robots
}

Authors: Thomas Linner, Wen Pan, Rongbo Hu, Charlie Zhao, Kepa Iturralde, Meysam Taghavi, Julian Trummer, Marcel Schlandt and, Thomas Bock

Chair for Building Realization and Robotics

Technische Universität München (TUM), Germany

\begin{abstract}
Purpose - Due to the sharply growing interest worldwide of "hard" physical-mechanical robot systems for the execution of on-site construction tasks (i.e. Single-Task Construction Robots, STCRs), development projects need a systematic design-management system to integrate the different needs and aims of stakeholders.
\end{abstract}

Research Methodology - This paper proposes a STCR Technology Management System (STCR-TMS) for the complete development cycle of STCR designs. The STCR-TMS is based on established principles from systems engineering and management and STCR-specific activities developed and tested by the authors as standalone elements in previous research work.

Findings - The application of the STCR-TMS revealed the practicability of the method and the underlying concepts to provide practical guidance for the development process. Additional findings indicate that the method is sufficiently generic and flexible for application to different types of robots and in different world regions. This research has also shown that key activities need to be addressed to increase the practicability of the STCR-TMS.

Originality/ Value - A unique characteristic of this method is the evolution with each utilization cycle. In addition, individual elements are interchangeable and can be adapted based on the external circumstances. These properties allow the TMS to be applied to other fields in construction robotics. With the progression of the verification and validation of the method, know-how and certain elements can be fed into standardization activities (e.g. establishing a Management System Standard (MSS)).

Keywords: Robotics, systems analysis and design, construction robotics, systems engineering, technology management, construction technology

Category: Technical paper

This article is (c) Emerald Group Publishing and permission has been granted for this version to appear here. Emerald does not grant permission for this article to be further copied/distributed or hosted elsewhere without the express permission from Emerald Group Publishing Limited.'

https://www.emerald.com/insight/content/doi/10.1108/CI-06-2019-0053/full/html

Citation

Linner, T., Pan, W., Hu, R., Zhao, C., Iturralde, K., Taghavi, M., Trummer, J., Schlandt, M. and Bock, T. (2019), "A technology management system for the development of single-task construction robots", Construction Innovation, Vol. ahead-of-print No. ahead-of-print. https://doi.org/10.1108/CI-06-2019-0053 


\section{Introduction}

For more than a decade, R\&D activities in on-site construction automation and robots were dominated by the development and integration of "soft" information-driven technology. Such technologies include Building Information Modelling (BIM) (Goulding et al., 2015), scheduling and construction process optimization techniques (e.g. Wang and Rezazadeh Azar, 2019), sensing systems (e.g. Kim et al., 2019) and Unmanned Aerial Vehicles (UAVs) (e.g. Zhou and Gheisari, 2018). As of recently, the development of "hard" physical-mechanical robots for the execution of specific on-site construction tasks (STCRs) have been gaining renewed interest. The interest manifests itself in increased academic research activity (Cai et al., 2018), joint industry-academia collaboration projects (e.g. Cordis, n.d.), and the emergence of start-ups (e.g. Bunkeberg Systems AB, n-Link, Fastbrick Robotics; O-Matic Intelligent Robot Ltd., Construction Robotics, Autonomous Solutions Inc., Kewazo, Built Robotics, Levaru, OutoBot, ROB Technologies, Tmsuk Co. Ltd., Constructions-3D, German Bionic, Okibo, etc.). Additionally, this has yielded a strong and growing interest of large, established organizations (Construction Industry Council Hong Kong, Excellence Group, Bouygues Construction, Thyssenkrupp, Country Garden Group, Hilti AG, Takenaka Corporation, Hitachi Construction, Hip Hing, Yau Lee, Züblin, etc.) towards the development of STCRs. A comprehensive overview of recent projects is given in Bock and Linner (2016).

STCRs are robots designed to execute a single task or multiple tasks on-site. Unlike more complicated methods, they do not try to automate or robotize major sections of the construction site, thereby avoiding large-scale investments or alterations. The development of construction robots involves diverse teams and stakeholders with backgrounds from the construction sector (contractors, developers, planners, engineering firms, etc.), from non-construction sectors (e.g. robotics, manufacturing, etc.), as well as investors. Therefore, the authors see the need to develop and evolve a generic design method, which allows stakeholders to streamline their activity, by integrating the different needs and objectives during system development. An efficient systematic cyclic design process with simple steps and tools can achieve this development.

\section{History and context of Singe-Task Construction Robots}

Based on a combined four decades of experience, the term STCR was first defined by Bock and Linner (2016), who sub-categorized the robots by typology, mechanical configurations, and task categories. The initial boom of STCRs originated from the Japanese construction industry during the 1970s. The trend moved towards integrated on-site factories in the 1980s (Linner, 2013), however global interest began to decrease, when the practical application revealed the immaturity of the systems.

Since the mid-2010s, applications such as energy-oriented renovation, asbestos removal or highrise construction, as well as significant advances in robot technology (e.g. in the automotive industry) led to a renewed interest in STCRs. Today, in many regions of the world, a mature robot component production infrastructure and company networks can manage system integration for location design and industry-specific manufacturing solutions. In addition, "soft" information driven infrastructures (i.e. BIM with integration of fabrication and machining knowledge see e.g. Hamid et al., 2018) have now matured to serve as an integrating backbone, thus allowing for the 
coordination of on-site robots with humans, other systems, logistics and supply, and overall onsite scheduling systems and processes. Additional facilitating preconditions are a more widespread use of prefabrication (e.g. Bock and Linner, 2015) and advances in material science (e.g. Bremner, 2008).

Nevertheless, a number of challenges remain. Particularly challenging are the significant differences between world regions (labor force, quality and safety standards, legal frameworks, materials, construction practices, stakeholder networks, supply infrastructure, access to technology, etc.) that demand individual, bottom-up solutions. This divide hinders the systematic build-up of knowledge and creates redundant development efforts. Additionally, it impedes the quick scaleup that investors demand.

\section{Scope and research question}

In this context, the authors focus on their recent research on developing and evolving a design process for STCRs. This process combines the needs and know-how of stakeholders, mitigates the aforementioned drawbacks, and considers best practices. The research question is how to facilitate and formalized the development procedure of STCRs, while considering the following issues: simplicity (i.e. low complexity, custom-made kinematic components, standard components, etc.), high scalability (i.e. easy adaptability to a range of tasks) and cost-effectiveness. An appropriate STCR development process needs to be generic in its nature and able to provide enough flexibility for different world regions, as well as different STCR task categories.

\section{Methodology: towards a management approach to develop construction robots}

Both systems engineering methods and management systems seek to provide generic best practice guidelines for the development, implementation, and improvement of new technology-based products and services. Systems engineering methods such as the V-Model approach (Firesmith, 2013) and the NASA Systems Engineering method (NASA, 2008) are usually of a generic and iterative type and applicable to a variety of tasks and industries. However, systems engineering methods specific to certain industries (e.g. Gausemeier et al., 2014; Holtmann et al., 2017) have also proven useful to integrate dedicated and domain-specific know-how. Newer approaches also address the increasingly multidisciplinary nature of systems, the need to systematically include stakeholders ( $\mathrm{Lu}$ et al., 2017) into the design process, and the need to manage continuous improvement (Fujimoto, 1999). Management systems usually complement systems engineering methods, have a broader orientation, and consider the organisation, business strategy, leadership, operation and performance measurement and improvement. The International Organization for Standardization (ISO) has developed a series of Management System Standards (MSS) from its generic MMS high level structure (ISO/IEC, 2010), such as the ISO/IEC 20000 standards series for service management (ISO, 2016, 2018).

Until now, only limited attempts have been made to explain the concepts and processes behind successful STCR developments. Bock (1988) introduced the concept of Robot-Orientated Design 
(ROD) for the parallel, harmonized development of the robot and its surroundings. Others reported on the subjects of modularization (e.g. Hasegawa et al., 1992), design and management tools (Bock and Linner, 2015), workflow diagrams and the design and optimization of the work progress (e.g. Kitahara et al., 2018). However, integrative and comprehensive guidance on how to systematically devise, strategically align, engineer requirements, design, develop, implement, evaluate, manage and, over time, mature STCR designs (i.e. their system architecture) and the respective Technology Readiness Levels is missing at present. In the view of the authors, this gap can be closed most efficiently by providing a practical, hybrid guidance system, situated between and seamlessly integrating systems engineering and management system methods - a so-called STCR Technology Management System (STCR-TMS).

The basic structure (Fehler! Verweisquelle konnte nicht gefunden werden.) follows the Deming cycle for continuous improvement (Richardson, 2010) (Layer 3). Flexible starting points and continuous improvement are crucial in the field of STCRs. Requirements, system architectures, work processes and business models are difficult to specify immediately, due to the lack of experience from long-term practical application. A cyclic and iterative approach is therefore pertinent. The core elements at the center are the four primary process steps (Layer 2). Each step comprises selected activities, tasks and outlined requirements (Layer 4). This is explained in detail in the subsequent sections of this paper. The sub-activities in the outer circle incorporate STCR-specific know-how that the authors gained through numerous STCR development projects, in which they were involved. The continuous evolution of the system architecture (ISO/IEC/IEE, 2011) and its TRLs (Layer 1) is the primary aim of the proposed process.

It is critical for any systems engineering or management approach to verify (initial formative feedback on the practical application) and validate (summative and quantitative feedback from large-scale application and testing) the method. Due to the lack of projects with long term and sustained market application of STCRs, a full-fledged validation of the STCR-TMS (i.e. statistical evaluation of application data or comparison of different development methods) is not feasible at this time. Nonetheless, the authors have verified elements and parts of the STCR-TMS, as well as their interaction in very recent development projects for construction robotics. The STCR-TMS represents an open and expandable system. The initial configuration will evolve over time, incorporating new experience and formative testing in future projects. 

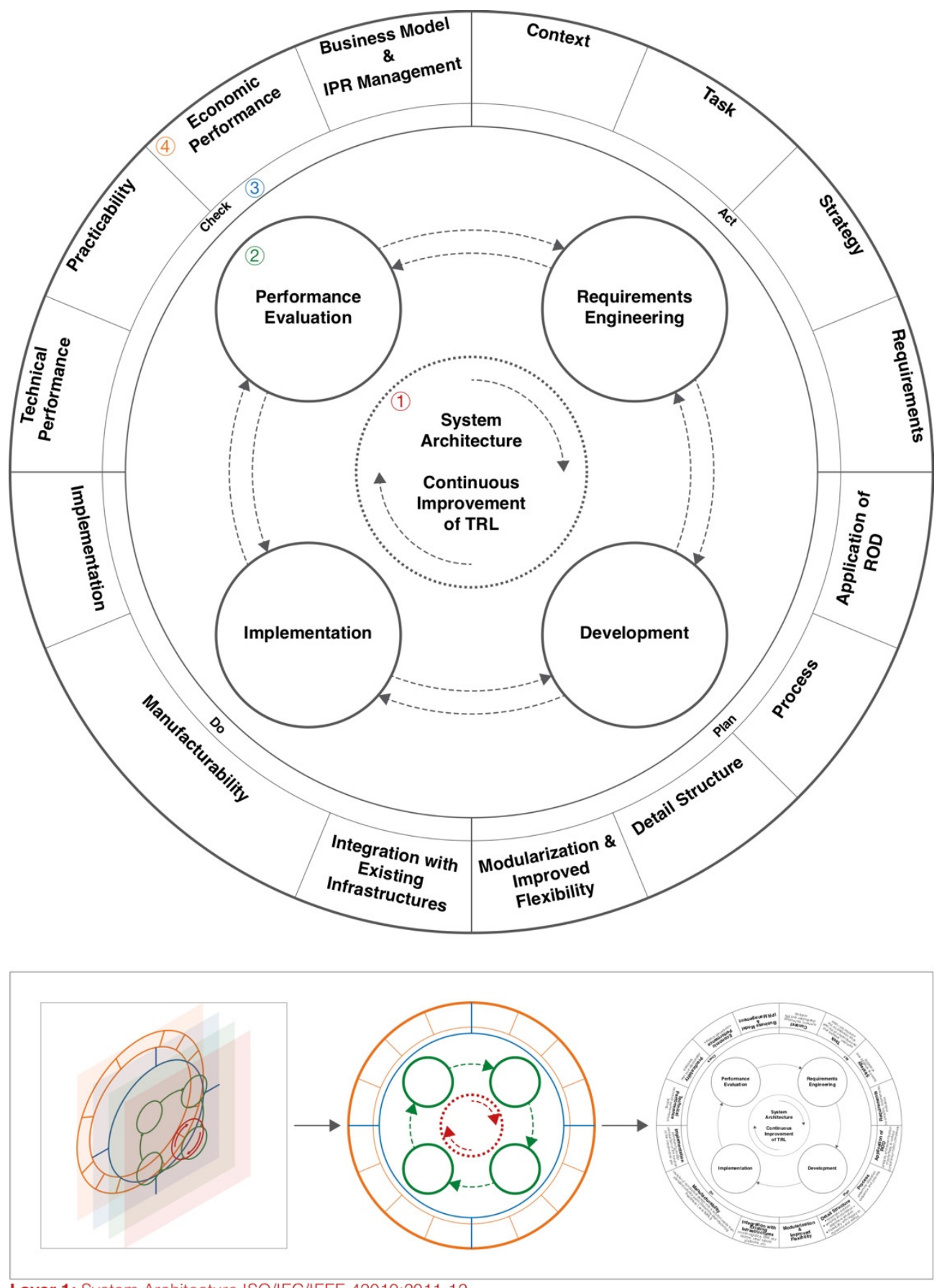

Layer 1: System Architecture ISO/IEC/IEEE 42010:2011-12

Layer 2: V-Model

Layer 3: Deming Cycle

Layer 4: STCR-specific elements

Figure 1: STCR Technology Management System (STCR-TMS). Image by the authors.

Following projects in which the authors were involved contributed to verify partially the cycle:

\section{- A façade processing robot for the Hong Kong CIC (CIC robot)}

The development of a façade processing robot for high-rise buildings in Hong Kong completed an initial STCR-TMS cycle. After implementing the first prototype, the follow-up project will allow for two additional run-throughs $\left(2^{\text {nd }}\right.$ cycle: extended prototype $\&$ improved 
business model; $3^{\text {rd }}$ cycle: fully functioning site-system $\&$ market introduction).

- A cable-driven panel installation robot in the project HEPHAESTUS

The development of a multifunctional end-effector for a robotic system for panel installation allowed for a verification of selected parts of the requirements engineering, development and implementation sections.

- Innovative façade renovation solutions in the project BERTIM

The development of automated and robotized solutions for the building renovation process with prefabricated modules allowed for a partial verification of the development and implementation sections (e.g. ROD, structure, integration with existing infrastructure, etc.).

- A scenario development technique for on-site construction robot technology

The development of a scenario technique for forecasting technology trends in specific construction industries in collaboration with HKU provided feedback for the initial phase of the requirement engineering section.

- Robotic solutions for elevator system installation by LEVARU

The start-up LEVARU is aiming to automate the process of aligning elevator guiderails during elevator installation. The development has run through several cycles. Feedback from industry insiders has led to a lower degree of automation to reduce development time, time to market, and risk.

The STCR-TMS, as depicted in Fehler! Verweisquelle konnte nicht gefunden werden., is composed of proven principles and methodologies from system engineering and management (Layers 1-3), which are combined with STCR-specific development concepts. The authors originally developed and tested the concepts represented in Layer 4 as stand-alone elements prior to the integration into the STCR-TMS (see e.g. Bock, 1988; Linner, 2013). Complete descriptions of the primary elements and their sub-activities and sub-concepts are in the following sections. The development process of the CIC robot serves as the primary example for explanation and verification.

\section{Requirements engineering (Act)}

This phase of the STCR-TMS aims to systematically establish the context and cornerstones for the development. This phase comprises four main steps: 1) analysis of the high-level context and industry trend of the use case; 2) streamlining the selection and decomposition of the concrete task for the robot; 3) development of a business strategy and stakeholder network; 4) defining the system requirements and performance indicators.

Context: scenario, technology, stakeholder and IPR analysis

First, the context within the industry use case setting needs to be analyzed (i.e. within the country and/or the region). The authors therefore developed a methodology to forecast technological trends and high-level requirements in a confined industry setting (Pan, Linner, et al., 2018) based 
on the scenario technique by Gausemeier et al. (1995). The forecasted trends can be used to focus the scope of the project (e.g. feasibility of different types of automation, etc.) and an initial highlevel analysis of stakeholders and the IPR context can be carried out. The authors have provided guidance on conducting stakeholder and IPR analysis prior to the development of robotic or mechatronic systems in Linner, Bekker, et al. (2017).

\section{Task: selection, analysis and decomposition of the task area foreseen for the construction robot}

Second, the robot's task or task category has to be carefully identified (see Bock and Linner, 2016). Among the activities during the development of the CIC robot was an online survey for previously identified stakeholders $(n=36)$ in combination with an in-depth process analysis on site i.e. workflow, techniques, regulations, interviews with workers and site managers, etc. $(t=4)$ (see Pan, Hu, et al., 2018).

\section{Strategy: business strategy and scalability}

Based on the selected task category, the initial business strategy can be developed. Identifying cost drivers in the conventional tasks execution and finding ways for efficient alterations for the robotic execution is key. At this point, the business strategy should also identify potentials for quick scale-up. Ideally, the selected task appears abundantly or frequently within the company or industry in question. The application scope can be increased by analyzing whether the selected task (or e.g. the kinematic motions) occurs similarly in other trades or even industries, in order to generate additional applications and market cases.

\section{Requirements: Co-creation, requirements, indicators}

A combination of the selected and analyzed task and the initial business strategy form the concrete use case, as well as the definition, detailing and prioritization of the requirements. Among the many possibilities to conduct a systematic requirements engineering process (see e.g. Pohl and Rupp, 2015), co-creation workshops with key stakeholders represent a straightforward way to define and prioritize the key system requirements. The practical application of the requirements co-creation method in the case of the CIC robot is described in Pan, Hu, et al. (2018). Translating the prioritized requirements in to system functionality requires measurable indicators. For the CIC robot, the first stage prototype was supposed to provide information regarding processing speed, cost, manufacturability, flexibility performance, as well as indications of possible challenges for future continuous on-site operation.

\section{Development sequence (Plan)}

This stage serves to define and detail the initial concrete model of the system architecture. The foremost important step is conducting a robot-oriented design (ROD) analysis in order to determine how complexity can be distributed between both the building system and the robotic system. Based on this, it is possible to develop the operation sequences and execution of the tasks in detail. 
Furthermore, the (kinematic) structure of the robot, as well as its modularity and flexibility can be determined and detailed.

\section{Application of Robot-Orientated Design (ROD)}

An in-depth ROD analysis is required in order to translate system requirements into concrete system functionality and design. This is necessary in order to determine how the complexity of the robot can be decreased by shifting some complexity to the overall system (e.g. the building system). For the CIC robot, the façades of the local public housing estates (primary use case) were thoroughly analyzed, which showed two findings: a) repeating façade elements can serve as reference points and as operation sequence blocks, and b) the necessary degrees of freedom could be significantly reduced with redesigned precast elements (Fehler! Verweisquelle konnte nicht gefunden werden.). Option "b" was withdrawn after consulting the stakeholders, because it needed adjustments throughout the value chain that would have been difficult to implement. In BERTIM, ROD was used to determine the necessary accuracy of the robot and the resulting tolerances of the building elements (Iturralde et al., 2017). 

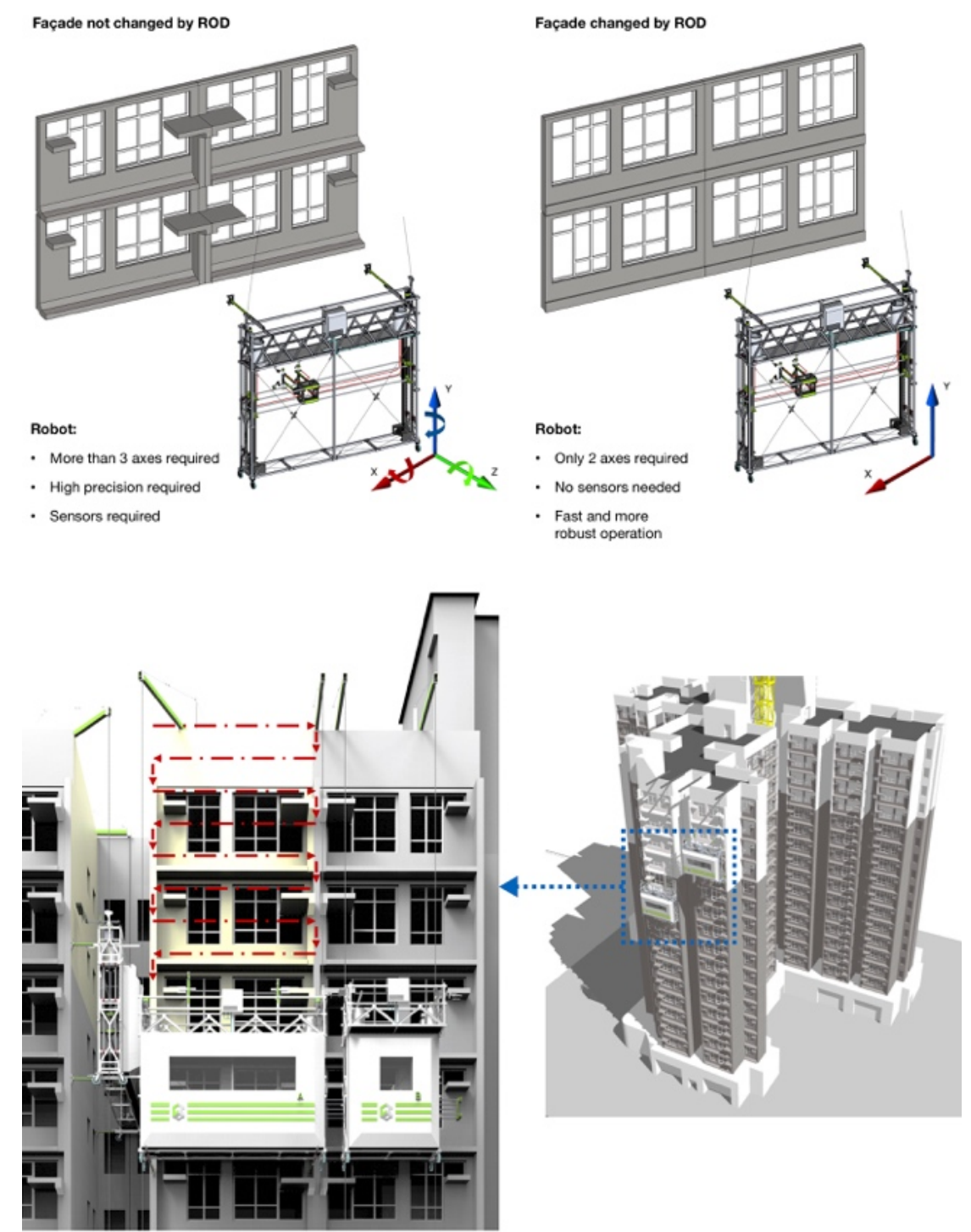

Proposed working process of the façade robot with painting function

Figure 2: top: ROD analysis for the CIC robot

bottom: Planning of the operations to determine the kinematic configuration

\section{Processes: determine operation sequences and processes}

Based on the results of ROD and the determined requirements, assembly line planning can follow. The term "assembly line" emphasizes that the construction process is a production process with the usual means of production: human resources, equipment, material, etc. Therefore, the following aspects must be considered when drafting the operation sequences and sub-activities of the robot and its surrounding elements: a) material flow of all involved elements; b) productivity focus according to previously specified performance indicators; c) ease of use and movement/logistics of the robot; d) degree of automation and human factors; e) implications across the value chain. 
In the case of the CIC robot, the operation sequence synchronized with the structure and shape of typical façade panels in Hong Kong public housing (Fehler! Verweisquelle konnte nicht gefunden werden.). All planned operations were represented in operation sequence diagrams. In Hephaestus, five different conceptual scenarios for the complex automatic installation process of façade elements by a cable-driven robot were introduced early on (Taghavi, Iturralde, et al., 2018).

\section{Detail structure of construction robot}

The physical-mechanical composition of the robot (i.e. basic kinematics) is a critical step in designing the system. The basic physical-mechanical sub-systems of an STCR are usually comprised of 1) the actual robot base body or frame, 2) the locomotion unit, 3) the end-effector, and 4) the material and tool supply system. For the CIC robot, the simplification of the operation sequence allowed the use of a simple gantry-type Cartesian system (see Fehler! Verweisquelle konnte nicht gefunden werden.), therefore avoiding the use of industrial robots. Reduced complexity means a significantly cheaper system.

\section{Modularization and flexibilization}

Modularity is one of the initial guiding principles when cycling through the STCR-TMS. After detailing the basic structure, the detailed concepts for modularity and flexibility of the robot system can follow development. The modularization of the base body, the tool change strategy and the modularity of the end-effectors are key in this context. The carrying frame (base body) of the CIC robot contains design features allowing easy adjustments to slightly varying heights and length of the façade panels. Deviating façade compositions (e.g. windows and cantilevering elements) were considered and are reflected in the design of the kinematic structure. Tool change allows the robot to be adapted for additional repetitive on-site tasks (i.e. different building types or in other industries such as shipbuilding). (see also Chair of Building Realization and Robotics, n.d.).

\section{Implementation and Prototyping (Do)}

After having detailed task sequence, structure, composition/design and modularity of the robot, the first implementation can be addressed. This phase of the STCR-TMS is usually resource- and time-intensive. It comprises detailing the integration with larger existing infrastructures, development towards higher Manufacturing Readiness Levels (MRLs) and the implementation either as a mock-up, a prototype or as the final product - depending on maturity and previous STCR-TMS cycles.

\section{Detailing of integration with existing infrastructures}

Ideally, a digital infrastructure allows the integration of STCRs into a digital twin of the construction site, simplifying scheduling, planning operations, programming movement and motion, etc. Digital surveys can provide information about the geometry and material composition of the environment, which the STCR requires to interact with the built environment. Algorithms can process the data to achieve spatial recognition. BERTIM showed that an STCR could operate even 
with little available data. For the CIC robot, the introduction of BIM as the local industry standard suggested to consider interoperability (Pan, Ilhan, et al., 2018). BIM could serve as the backbone for an integrated service platform (ISP) for STCRs.

\section{Manufacturability:}

The design generated in previous STCR-TMS cycles shall be suitable for a cost-efficient production. This requires determining the MRL. The necessary resources and the cost of the robot should be minimized, while ensuring robustness for on-site operation. Cost can be reduced significantly by utilizing mostly linear axes or other cheap standard automation components and avoiding the integration of costly types of industrial robots - exceptions are possible.

The operation sequence of the CIC robot was simplified to an extent, which allowed it to be built entirely from standard linear axes and drives (Figure 3 top), making the Bill of Materials (BOM) more cost-efficient and facilitates the setup of a manufacturing system for the robot. In HEPHAESTUS, a hybrid "macro/micro" positioning approach was selected (Figure 3 bottom). A simple, cost-effective cable robot system serves as the macro kinematic structure, while a light end-effector system with a small industrial robot handles fine positioning (Taghavi, Heredia, et al., 2018). 

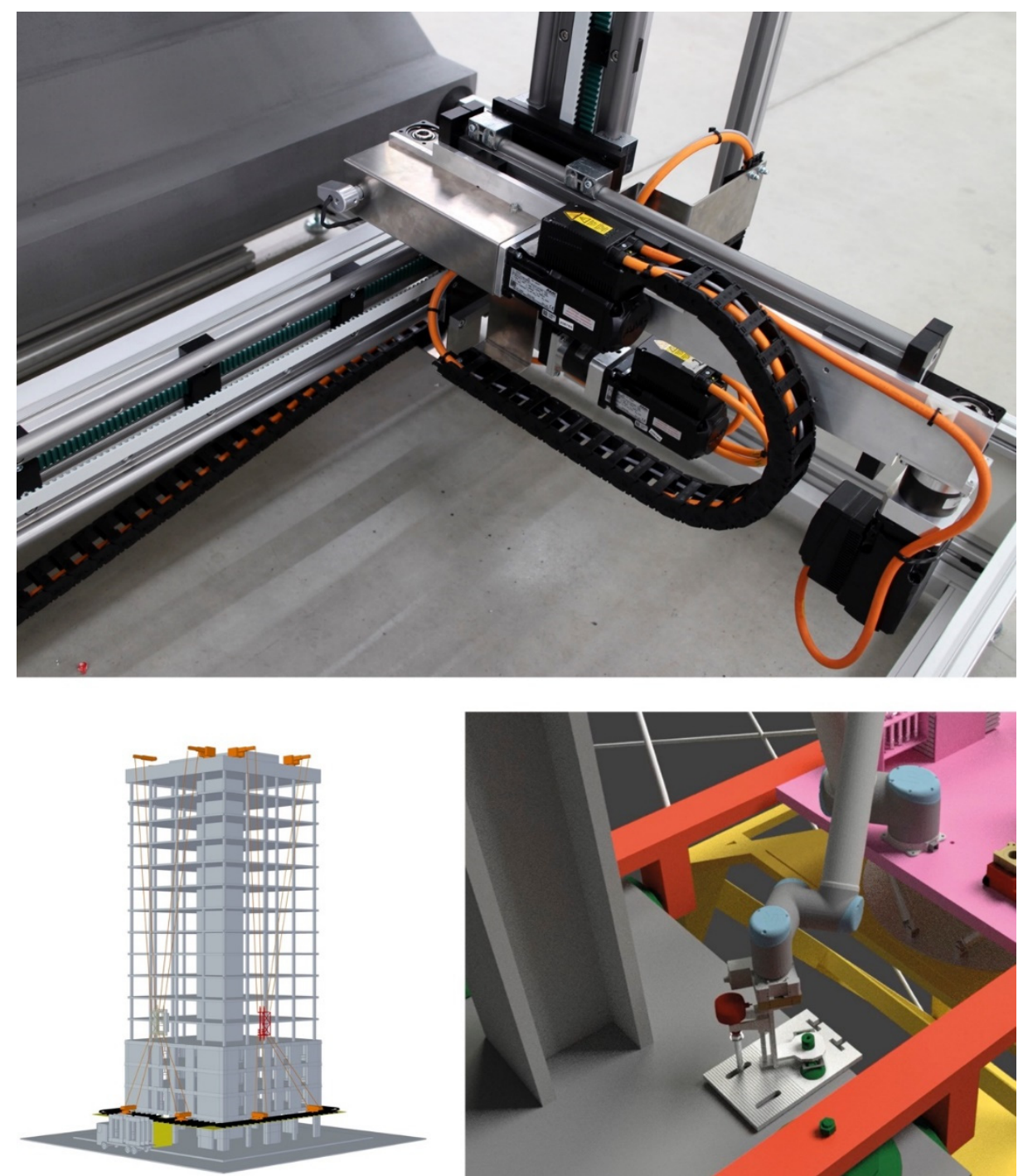

Figure 3: top: The CIC robot is exclusively composed of standard linear axes and drives. bottom: In HEPHAESTUS, the main kinematic structure consists of a cable robot system, while an industrial robot is utilized for the end-effector.

\section{Implementation}

Due to limited experience with practical applications of STCRs, iterative prototyping and testing is crucial. It is important to include information from stakeholders at all levels (workers, site managers, contractors, robot system suppliers, the public, etc.). The initial prototyping should primarily focus on technical performance. 

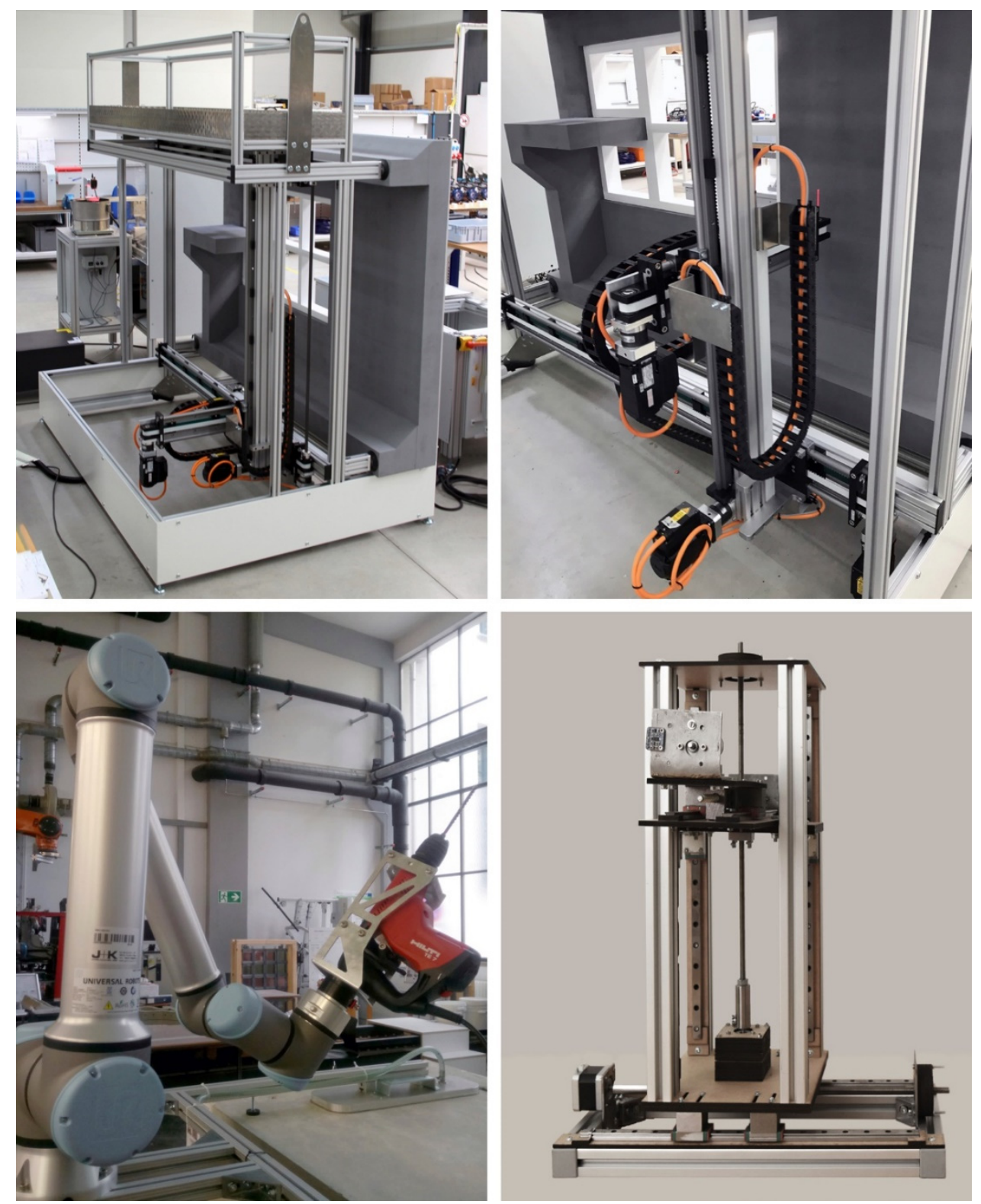

Figure 4: Top left and right: prototype of the CIC robot. bottom left: prototype developed in HAEPHAESTUS. bottom right: LEVARU prototype

Concepts such as TRL allow for valid feedback from users and potential interference with the onsite ecosystem in later stages. According to the feedback, the following phases have to be completed before the final "mission ready" system: laboratory testing, testing in relevant/simulated environment and testing in real world environment. These key steps in the development process are reflected in different prototypes in the example projects. The prototype of the CIC robot was designed to yield information on processing speed, system flexibility and system cost, as well as feedback from manufacturers and contractors. In HEPHAESTUS, each subsystem is implemented individually at first, followed by the integration and testing of the entire system. For LEVARU, the robot system was first prototyped as a 1:2 scale mock-up, before the concept was altered to a human-guided on-site assistive device and subsequently prototyped, reaching TRL 4. In BERTIM, early implementation phases revealed unexpected problems with major deviations in the grasping process, which were addressed with the introduction of additional sensing systems (Iturralde et al., 2019). 


\section{Performance evaluation (Check)}

In order to start the next STCR-TMS cycle, the system must be evaluated against the performance indicators from the requirements engineering phase. Prototype and study design should be developed in conjunction. Performance indicators should be tailored to the specific project, available resources and desired TRL. Three performance categories are key for STCRs: technical performance, practicability/usability, and economic performance. Based on this, it is possible to further develop the business model and intellectual property (IP) strategy.

\section{Proof of concept: laboratory testing and public demonstration}

The prototype of the CIC robot was tested in the laboratory to verify the concept, kinematics and functionality of the robot. The end-effector is able to cover the majority of the façade surface and can be swiftly exchanged. In simulations (with a laser beam instead of paint), the 1:2 scale model of a façade panel could be painted in 150 seconds. Prototyping cost provided data for future costbenefit calculations. Additionally, the weight of the robot will have to be reduced in future development in order to decrease the load on the building and hoisting system. The exhibition and demonstration of the prototype at the Construction Innovation and Technology Application Centre (CITAC) in Hong Kong stimulated exchange with engineers and professionals and raised public awareness for construction robotics.

\section{Practicability and usability: stakeholder and user feedback (Co-creation 2)}

Besides the technical performance, it is important to evaluate the practicability and usability performance of the system. The development team of the CIC robot carried out initial explorative usability testing in the laboratory. Testing revealed that, for example, the calibration of the robot after emergency stops might cause severe maintenance issues in real-world operation. During the exhibition at the CITAC, additional explorative feedback from stakeholders and potential investors could be acquired, thereby serving as a second co-creation phase in which practicability and usability requirements can be refined.

\section{Economic performance: Cost Benefit Analysis (CBA)}

Both CBA and cost-effectiveness analysis (CEA) are useful tools to measure, evaluate the investment potential, and can guide the decision-making process of investors. While the CBA is looking at the monetary aspects, the CEA focuses on non-monetary outcomes such as incremental cost per unit of effectiveness. Conducting a CEA first can lay a foundation for CBA calculation in later stages (Warszawski and Rosenfeld, 1994).

Many non-quantitative terms are yet to be defined in the context of the CIC robot. Guided by the prototyping cost, a value-based price method is used to estimate the Net Present Value (NPV) costs and benefits of the system. The rest of the cost data was collected in close collaboration with the contractor (Hip Hing) and the Hong Kong Polytechnic University (PolyU). The detailed structure and the outcomes of the authors' method will be presented in an upcoming publication. 


\section{Business model \& IPR management}

The first STCR-TMS cycle for the CIC robot helped to clarify the relations of the involved stakeholders and to determine whether contractors will purchase the system from a third-party system supplier or integrate it into their company, for example. Various stakeholders such as contractors, robot system manufacturers and developers qualify as investors. Once CIC saw the emergence of a team of stakeholders to jointly invest in a second STCR-TMS cycle, CIC in their role as a construction industry association pursued the filing of a patent in order to be able to license it in a facilitating manner to the co-investors for cycle 2 and/or other players in the future.

\section{Discussion and outlook}

The aforementioned projects showed that the STCR-TMS and its underlying principles provide comprehensive and practical guidance on concrete steps forward. Moreover, the application of the STCR-TMS in the aforementioned projects showed two important findings. The scheme is sufficiently flexible and generic to be applicable to different robot types (e.g. CIC robot, LEVARU, etc.) and in different world regions (Hong Kong, EU, etc.). In particular, the STCRTMS accelerated the development process in the more market-driven initiatives such as the CIC robot development project. Less time was spent deciding the development measures and stakeholders and coordinating them.

\section{Feedback and lessons from the application of the STCR-TMS:}

Overall, the following feedback and lessons were extracted from the application of the STCRTMS in the above outlined projects:

1. Use TRLs and system architecture descriptions: Continuous updates of the TRLs of subsystems and technology and a schematic representation of the system architecture proved practical to assess the project maturity and potential risks (e.g. estimates on needed resources and time needed for the development). For future use, integrating a risk assessment procedure into the STCR-TMS may be useful. The authors' projects show that it is feasible to further the TRL by 1-2 levels per cycle.

2. Modularity enables scalability: Early consideration of modularity on basic system levels (end-effectors, base body, sensors, etc.) allows for planned faster scalability and flexibility. This can lower the technological risks for system manufacturers and investors by determining and adjusting the tasks and task range. A modular design allows adapting to different tasks and end-effectors, as well as changing business models in the long term.

3. ROD - be aware of the trade-offs: ROD has the potential to lower complexity and cost across the value chain. However, with the current structure of the construction industry, this is difficult to achieve. The CIC robot could operate without electronic sensor systems, if reference elements were integrated in the precast façade elements. However, the required effort 
for key stakeholders outweighed the prospective performance increase. The trade-offs of shifting complexity between robot and building system have to be balanced carefully.

5. Do not underestimate human factors: The robot has to be designed with the current skillsets of construction trades in mind, since specially trained operators do not exist. The acceptance and ease of use by the end-user determines ultimately the efficiency and success of the system.

6. Performance quantification is key: A CBA is crucial to cross the line from research to more business-orientated development cycles and can help to incentivize stakeholders to sustain their investment. The implementation step should be used to feed the economic calculation with data in a bottom-up approach.

7. Reduce technical complexity: The robot solution needs to be simple, robust and cost efficient. Therefore, the necessary DOFs of the system, as well as complex maneuvers and localization tasks should be kept to a minimum to be able to work with linear axes and standard components as much as possible.

8. Consider market access early on: Consider the local market structure. While typical robot manufacturers might be reluctant to invest or lack market access, contractors or construction equipment manufacturers do not master STCR technology and have difficulties to assess both complexity and risk. In addition, interdisciplinary alliances are often not easy to manage and may complicate IPR ownership.

9. Consider standardization and certification: Experience shows that a key barrier to investment is uncertainty regarding the performance, the involved work processes, required skill levels and proof of impact. An elaborated and a commonly agreed upon set of criteria (for the local industry) for the robot and the development process can reduce these risks and facilitate efficient decision-making.

\section{Next systematic steps to evolve the TMS}

Based on the previously outlined feedback and lessons, the authors developed a future roadmap for the expansion of the STCR-TMS method (Figure 5). First, it is necessary to modularize and create new interchangeable Level 4 elements (depending on region, TRL, etc.). Second, key Level 4 activities can be developed further as part of a "toolkit approach", speeding up development and ensuring a level of quality. Third, modularity allows creating TMS instances for other construction robotics areas (e.g. on-site factories, infrastructure, etc.). Fourth, in parallel with more experience, it will be possible to make the transition from verification to validation (e.g. by more comprehensively comparing the performance of development teams using the TMS). Finally, it will be possible to create generally recognized standards for construction robotics. 


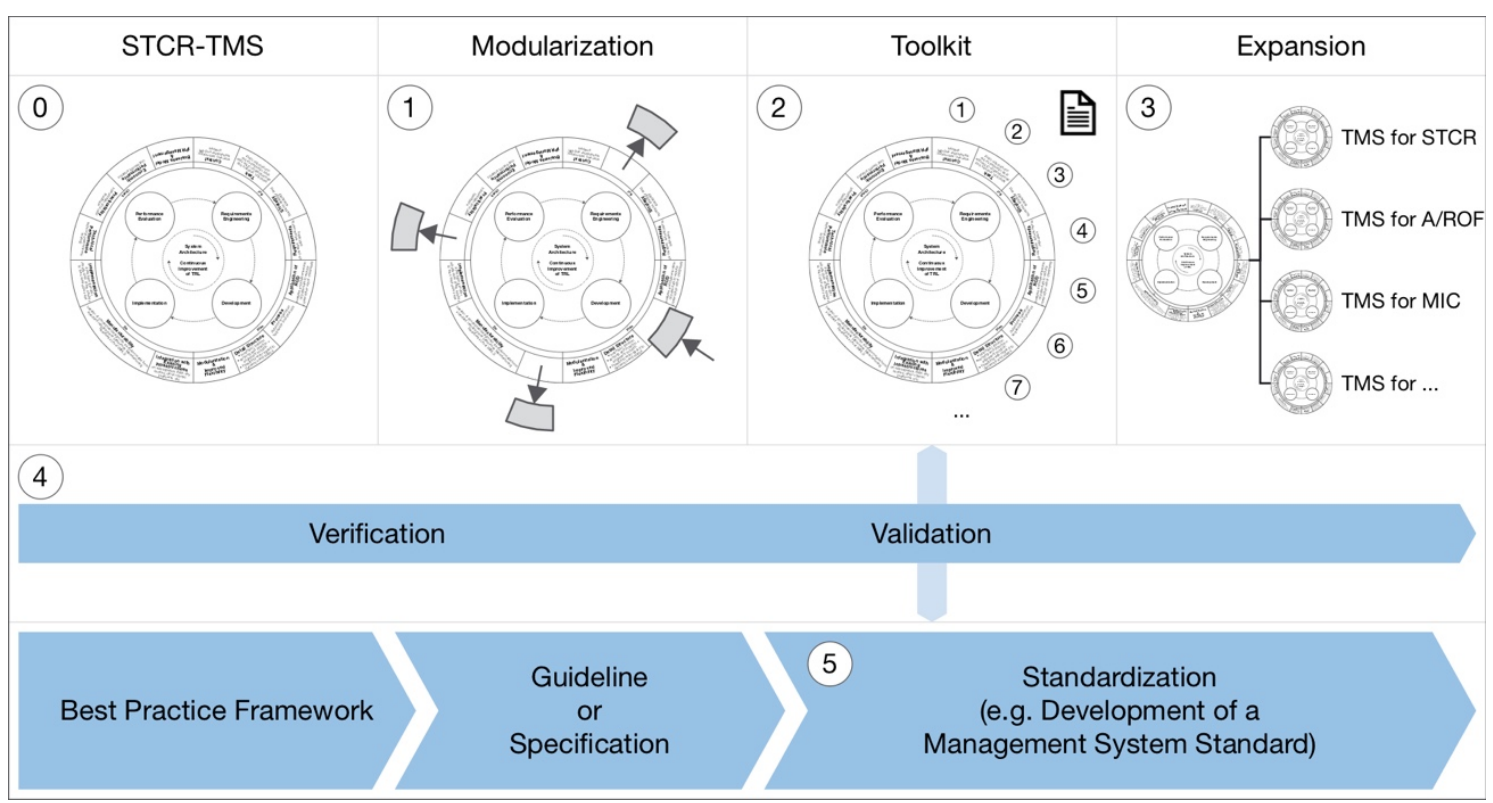

Figure 5: Towards modularization, provision of a toolkit, expansion and standardization based on the authors' framework.

\section{Conclusion}

A STCR Technology Management System (STCR-TMS) was proposed as an integrative and comprehensive guidance on how to systematically conceptualize, strategically align, engineer requirements, design, develop, implement, evaluate, manage, and over time, mature STCR designs. The authors have used five recent and ongoing projects to test and verify parts of the proposed cyclic method. The STCR-TMS is composed of an adapted set of proven principles and methodologies from the systems engineering and management field, combined with set of activities enlisting concepts specific for STCR development, which had already been tested by the authors in previous work. The application of the STCR-TMS revealed that the method and its underlying concepts are feasible providing comprehensive and practical guidance. The market access strategy should be considered early on in the development process. Performance quantification methods such as a CBA are crucial tools. Reduced technical complexity and scalability through modularity are also key. Most importantly, the efficiency of the development process could be increased by utilizing TRLs and system architecture descriptions. The application also revealed critical key activities that need to be addressed specifically and in more detail in improved versions to further increase the practicability of the STCR-TMS. It was shown that a unique characteristic of the proposed STCR-TMS method is that its core is built from modular principles that allow it to be evolved over time with each cycle of use. With increased use and the advancement of verification and validation over time, the basis will be built for the creation of a Management System Standard (MSS). 


\section{Acknowledgments}

This research was supported and partly financed by the following entities and projects:

1 Development of a façade processing robot for CIC. This project was commissioned by the Construction Industry Council Hong Kong.

2 Development of a cable driven panel installation robot in the project HEPHAESTUS: this project has received funding from the European Union's H2020 Programme (H2020/2014-2020) under Grant Agreement Number 732513

3 Development of innovative façade renovation solutions in the project BERTIM: this project has received funding from the European Union's H2020 Programme under Grant Agreement Number 636984

4 Development of a scenario development technique for on-site construction robot technology: this project has received funding from the German Academic Exchange Service (DAAD Grant No. 57217359) and the Research Grants Council of Hong Kong (Reference No. G-HKU704/15).

5 Development of robotic solutions for elevator system installation by LEVARU: the LEV RU authors thank the LEVARU team for their kind cooperation.

\section{References}

Bock, T. (1988), Study on Robot-Oriented Construction and Building System, Dr.-Ing. Dissertation, The University of Tokyo, Tokyo, Japan.

Bock, T. and Linner, T. (2015), Robot-Oriented Design: Design and Management Tools for the Deployment of Automation and Robotics in Construction, Vol. 1, Cambridge University Press, Cambridge.

Bock, T. and Linner, T. (2016), Construction Robots: Elementary Technologies and Single-Task Construction Robots, Vol. 3, Cambridge University Press, Cambridge, UK.

Bremner, T.W. (2008), "8 - Lightweight concrete", in Mindess, S. (Ed.), Developments in the Formulation and Reinforcement of Concrete, Woodhead Publishing, pp. 167-186.

Cai, S., Ma, Z., Skibniewski, M., Guo, J. and Yun, L. (2018), “Application of Automation and Robotics Technology in High-Rise Building Construction: An Overview”, 2018 Proceedings of the 34th International Symposium on Automation and Robotics in Construction, Berlin, Germany, pp. 309-316.

Chair of Building Realization and Robotics. (n.d.). "Development of a Multifunctional Facade and Exterior Finishing Robot for The Construction Industry Council (CIC) in Hong Kong”, available at: http://www.br2.ar.tum.de/index.php/component/content/article?id=352 (accessed 1 September 2019).

Cordis. (n.d.). "Highly automatEd PHysical Achievements and PerformancES using cable roboTs Unique Systems", Cordis EU Research Results, available at: https://cordis.europa.eu/project/rcn/206251/results/en (accessed 23 May 2019).

Crowder, J.A., Carbone, J.N. and Demijohn, R. (2016), Multidisciplinary Systems Engineering: Architecting the Design Process, Springer International Publishing, Cham, Germany. 
Firesmith, D. (2013), "Using V Models for Testing”, Carnegie Mellon University, Software Engineering Institute $\mathrm{Blog}, \quad, 11$ November, available at: https://insights.sei.cmu.edu/sei_blog/2013/11/using-v-models-for-testing.html (accessed 22 May 2019).

Fujimoto, T. (1999), The Evolution of a Manufacturing System at Toyota, Oxford University Press, New York, NY, USA.

Gausemeier, J., Fink, A. and Schlake, O. (1995), Szenario-Management: Planen und führen mit Szenarien, Hanser, Munich, Germany.

Goulding, J., Pour Rahimian, F., Abrishami, S. and Ganah, A. (2015), "Virtual generative BIM workspace for maximising AEC conceptual design innovation: A paradigm of future opportunities", Construction Innovation, Vol. 15 No. 1, pp. 24-41.

Hamid, M., Tolba, O. and El Antably, A. (2018), "BIM semantics for digital fabrication: A knowledge-based approach", Automation in Construction, Vol. 91, pp. 62-82.

Hasegawa, Y., Onoda, T. and Tamaki, K. (1992), "Modular robotic application for flexible building construction", Proceedings of 13th International Symposium on Automation and Robotics in Construction, Japan International Association for Automation and Robotics in Construction, Tokyo, Japan, pp. 113-122.

ISO. (2016), "Medical devices -- Quality management systems -- Requirements for regulatory purposes", ISO 13485:2016, available at: http://www.iso.org/cms/render/live/en/sites/isoorg/contents/data/standard/05/97/59752.html (accessed 1 September 2019).

ISO. (2018), "Information technology -- Service management -- Part 1: Service management system requirements", ISO/IEC 20000-1:2018, available at: http://www.iso.org/cms/render/live/en/sites/isoorg/contents/data/standard/07/06/70636.html (accessed 1 September 2019).

ISO/IEC. (2010), "ISO/IEC Directives, Part 1 - Procedures for the Technical Work, Annex L (Normative) - Proposals for Management System Standards", available at: https://www.iso.org/sites/directives/current/consolidated/index.xhtml (accessed 1 September 2019).

ISO/IEC/IEE. (2011), "Systems and software engineering -- Architecture description", ISO/IEC/IEEE 42010:2011, available at: http://www.iso.org/cms/render/live/en/sites/isoorg/contents/data/standard/05/05/50508.html (accessed 1 September 2019).

Iturralde, K., Linner, T. and Bock, T. (2017), "First Monitoring and Analysis of the Manufacturing and Installation Process of Timber Based 2D Modules for Accomplishing a Future Robotic Building Envelope Upgrading", 2017 Proceedings of the 34th ISARC, Taipei, Taiwan, pp. 65-73.

Kim, M.-K., Wang, Q. and Li, H. (2019), "Non-contact sensing based geometric quality assessment of buildings and civil structures: A review", Automation in Construction, Vol. 100, pp. 163-179.

Kitahara, T., Satou, K. and Onodera, J. (2018), "Marking Robot in Cooperation with Three-Dimensional Measuring Instruments", 2018 Proceedings of the 35th ISARC, Berlin, Germany, pp. 292-299. 
Linner, T. (2013), Automated and Robotic Construction: Integrated Automated Construction Sites, Dr.-Ing. Dissertation, Technical University of Munich, Munich, Germany.

Linner, T., Bekker, M.M., Groth, A., Lu, Y., Solcanu, G., Steinhardt, E. and Valk, C.A.L. (2017), IPR Management - Outline of the Code of Conduct Related to the Management of Intellectual Property Rights (IPRs) in Order to Ensure an Open Atmosphere within the Consortium as Well as to Foster Efficient and Fast Exploitation of Project Results, available at: http://reach2020.eu (accessed 31 January 2017).

Lu, Y., Valk, C.A.L., Steenbakkers, J.J.H., Bekker, M.M., Visser, T., Proctor, G.M., Toshniwal, O., et al. (2017), "Can technology adoption for older adults be co-created?", Gerontechnology, Vol. 16, pp. 151-159.

NASA. (2008), NASA Systems Engineering Handbook., United States Government Printing Office, Washington, USA.

Pan, M., Linner, T., Pan, W., Cheng, H. and Bock, T. (2018), "A framework of indicators for assessing construction automation and robotics in the sustainability context", Journal of Cleaner Production, Vol. 182, pp. 82-95.

Pan, W., Hu, R., Linner, T. and Bock, T. (2018), “A Methodological Approach to Implement OnSite Construction Robotics and Automation: A Case in Hong Kong”, 2018 Proceedings of the 35th ISARC, Berlin, Germany, pp. 362-369.

Pan, W., Ilhan, B. and Bock, T. (2018), "Process Information Modelling (PIM) Concept for Onsite Construction Management: Hong Kong Case", Periodica Polytechnica Architecture, Vol. 49 No. 2.

Pohl, K. and Rupp, C. (2015), Requirements Engineering Fundamentals: A Study Guide for the Certified Professional for Requirements Engineering Exam, Foundation Level, IREB Compliant, Second edition., Rocky Nook, Santa Barbara, CA, USA.

Richardson, G.L. (2010), Project Management Theory and Practice, CRC Press, Boca Raton, FL, USA.

Taghavi, M., Heredia, H., Iturralde, K., Halvorsen, H. and Bock, T. (2018), "Development of a Modular End Effector for the installation of Curtain Walls with cable-robots", Journal of Facade Design and Engineering, Vol. 6 No. 2, pp. 1-8.

Taghavi, M., Iturralde, K. and Bock, T. (2018), "Cable-Driven Parallel Robot for Curtain Wall Modules Automatic Installation", 2018 Proceedings of the 35th ISARC, Berlin, Germany, pp. 396-403.

Wang, Z. and Rezazadeh Azar, E. (2019), "BIM-based draft schedule generation in reinforced concrete-framed buildings", Construction Innovation, Vol. 19 No. 2, pp. 280-294.

Warszawski, A. and Rosenfeld, Y. (1994), "Robot for Interior-Finishing Works in Building: Feasibility Analysis", Journal of Construction Engineering and Management, Vol. 120 No. 1, pp. 132-151.

Zhou, S. and Gheisari, M. (2018), "Unmanned aerial system applications in construction: a systematic review", Construction Innovation, Vol. 18 No. 4, pp. 453-468. 